\title{
ON THE GYROSCOPE
}

\author{
BY
}

WILLIAM F. OSGOOD

\section{The Problem and its Solution}

The problem which led to the results of this paper was the following.* Consider a gyroscope $f$ which is rotating with high velocity about its axis, the center of gravity, $O$, being fixed in space. Let a couple, $\mathfrak{M}$, be applied. The axis of the gyroscope will in general describe a cone, $\Re$, whose vertex is at $O$. What is the relation between the geometric configuration (i.e., the cone $\Re$ ), the velocity with which the axis sweeps it out, and the couple?

Instead of the center of gravity, any other point $O$ of the axis may be chosen as the point of the body which is to be fixed in space. Let any forces be applied, and consider the vector moment of each with respect to $O$. The sum of these vectors shall be denoted by $\mathfrak{M}$, and the question in its new formulation is clear.

Again, let the gyroscope be free to move in space under the action of any forces. Its rotation is the same as that of an equivalent gyroscope whose center of gravity is held fast, and which is acted on by the same relative forces. The motion of the latter gyroscope is precisely the case discussed here.

* Since returning the galleys my attention has been called to Lamb, Higher Mechanics, 1920, p. 133, where the formulas appear: $A v d \chi / d t=Q+C n v, A d v / d t=P$. Mr. Lamb obtains a highly interesting result by interpreting these equations in terms of the motion of a particle of mass $A$ constrained to slide on a smooth sphere and acted on by a force tangent to the sphere, whose components are $P$ and $Q$, and in addition by a force along the normal to the path, equal to $C n v$. He does not, however, make the cone $\Omega$ of the present article an element of his discussion, and so of course does not introduce the conception of the bending of this cone; nor does he mention the geodesic curvature of the curve we have called $\mathfrak{E}$. Moreover, in his discussion of the rising of the axis of the top with blunt peg, spinning on a rough table, he arrives at the usual false result (p. 136) that friction always causes the axis to rise; cf. $\$ 6$ of the present article. The present paper was sent to the $\mathrm{Tr}$ a n s a c $\mathrm{t}$ i o $\mathrm{n}$ s on March 19, 1921, and presented to the Society at its April meeting, 1921; cf. B u 11 e $t$ i n of the American Mathematical Society, vol. 27 (1921), p. 391.

$\dagger$ By a gyroscope we mean a rigid body having an axis of material symmetry. More precisely, there is a line in the body, called the axis, which has the following properties: (i) it passes through the center of gravity; (ii) if $O$ is any point of this line, the latter coincides with one of the principal axes of inertia through $O$; denote the moment of inertia about this axis by $C$; (iii) the moments of inertia about all other principal axes through $O$ are equal: denote their common value by $A$; (iv) $A$ and $C$ are both positive. 
Let $\zeta$ be a point of intersection of the axis of the gyroscope with the unit sphere about $O$ as center; and let $\zeta$ be so chosen that an observer at $O$, looking toward $\zeta$, would see the gyroscope rotating in the positive sense, which we shall take as counter-clockwise. Let $\sqrt{ }$ be the curve on the unit sphere traced out by $\zeta$. It may be regarded as the directrix of the cone $\Omega$.

We next introduce a measure of the bending of the cone $\Omega$. Suppose that, fixing our attention on an arbitrary cone $\Omega$ with its vertex at $O$, we allow $\zeta$, the point in which a generating ray meets the unit sphere, to describe its path $\mathbb{S}$ with unit velocity. Then the rate at which the tangent plane to $\Omega$ at $\zeta$ is turning shall be defined as the bending of the cone, and denoted by $\kappa$.

In other words, let $P$ be an arbitrary point of $\left(\mathcal{S}\right.$, and $P^{\prime}$ a neighboring point. Let $\epsilon$ denote the angle between the tangent planes at $P$ and $P^{\prime}$. Then

$$
\kappa=\lim _{P^{\prime} \rightarrow P} \frac{\epsilon}{P^{\prime}} .
$$

The angle $\epsilon$ is also the angle between the normals to the tangent planes. Denote the point of intersection of such a normal, thought of as directed and drawn from $O$, with the unit sphere by $Q$. Then $\kappa$ is the rate at which $Q$ is moving when $P$ moves with unit velocity.

It is convenient, however, to introduce $\kappa$ as an algebraic quantity. Let $s$ be so chosen that, as $\zeta$ describes $\mathfrak{E}$, $s$ increases. Then $v=d s / d t$ is positive. The tangent to $\mathbb{S}$ in the sense of the increasing $s$ shall be called the positive tangent, and the normal drawn to the right in the tangent plane of the sphere at $P$, when the observer, outside the sphere, is walking along $\mathbb{f}$ in the positive sense, the positive normal;* cf. Fig. 1. And now $\kappa$ shall be taken as positive when $\mathbb{5}$ near $P$ lies to the left of the great circle arc which is tangent to $\mathbb{E}$ at $P$, i. e., on the side of the negative normal; and as negative, when the opposite is the case.

The concept of the bending, $\alpha$, is akin to that of the curvature of the cone $\Omega$, thought of as the one-dimensional locus of its generators. But this idea of curvature has nothing to do with the curvature of $\Omega$, when thought of as a two-dimensional manifold.

The bending, $\kappa$, of $\Omega$ is identical (save, possibly, as to sign) with the geodesic curvature of $\mathfrak{E}$, regarded as a curve on the sphere. Moreover, $\kappa$ is connected with the curvature, $K$, of $\mathfrak{C}$, regarded as a space curve, by the relation:

$$
\kappa^{2}=K^{2}-1 \text {. }
$$

Finally, $\alpha$ is related to the torsion, $T$, of $\subseteq$ by the equation $\dagger$

* More precisely, the positive normal at $P$ is oriented with respect to the positive tangent and the direction of $O P$ as the positive $y$-axis is oriented with respect to the positive axes of $x$ and $z$.

† This latter relation was pointed out to me by Professor C. N. Haskins, to whom I am indebted for a careful examination of this paper, and for valuable suggestions. 


$$
\frac{d}{d s} \tan ^{-1} \kappa= \pm T
$$

A case of great interest is the restricted one in which $\mathfrak{M}$ is perpendicular to the axis of the gyroscope.* Here, the component couple tending to produce rotation about the axis is nil, and hence the component angular velocity $r$ about this axis $\dagger$ is constant throughout the motion; denote it by $\nu: r=\nu$.

We are now prepared to state our conclusion in the restricted case.

THEOREM I. The relation which holds between $\kappa, v$, and $\mathfrak{F}$ is the following:

$$
\begin{gathered}
A v \frac{d v}{d s}=T, \\
A \kappa v^{2}+C \nu v=Q,
\end{gathered}
$$

where $T$ and $Q$ denote respectively the components of $\mathfrak{F}$ along the positive tangent and the negative normal of $\mathbb{5}$ at $\zeta$.

This theorem explains in part a vagueness of expression and ideas frequently met in discussions of the gyroscope, for questions are often asked, and answers given, both of which must, to have any meaning, involve all three quantities, $\kappa, v$, and $\mathfrak{F}$; but one is omitted. Thus, for example, the statement often made that "when a couple is applied to a rotating gyroscope, the forces of the couple intersecting the axis of the gyroscope at right angles, the axis will move in a plane perpendicular to the plane of the forces of the couple" is false. In fact, the axis will begin to move tangentially to this plane, if it starts from rest, $\ddagger$ and all intermediate cases are possible, according to the initial motion of the axis.

The general case is that in which $\mathfrak{M}$ is arbitrary, $O$ being fixed. Let the component of $\mathfrak{M}$ along $O \zeta$ be denoted by $N$. The other component of $\mathfrak{M}$, i. e., the component perpendicular to $O \zeta$, is dynamically equivalent to a force $\mathfrak{F}$ at $\zeta$ perpendicular to $O \zeta$ and equal numerically to this component. Let the components of $\mathfrak{F}$ along the positive tangent and negative normal of $\mathbb{S}$ be denoted as before by $T$ and $Q$. Then the complete statement of the dynamical conditions governing the motion is contained in the following theorem.

THEOREM II. The three dynamical equations governing the motion of the gyroscope can be written as follows:

* When $\mathfrak{M}$ is thought of as a couple, the vector $\mathfrak{M}$ is a vector at right angles to the plane of the couple, its length and sense heing determined in the usual manner.

$\dagger \mathrm{By}$ this is meant that the vector angular velocity about the instantaneous axis is resolved along three mutually perpendicular directions, one of which is the axis of the gyroscope; more precisely, the ray $O \zeta$. Moreover, the forces acting on the gyroscope may be replaced by a single force $\mathfrak{F}$ at $\zeta$ perpendicular to the axis, and the reaction at $O$. The plane of the couple $\mathfrak{M}$ is determined by $\mathfrak{F}$ and the point $O$, and the magnitude of $\mathfrak{M}$ is equal to that of $\mathfrak{F}$.

$\ddagger$ The ordinary top with fixed peg is a case in point, if the top is released with the axis at rest. 


$$
\left\{\begin{array}{c}
A v \frac{d v}{d s}=T, \\
A \kappa v^{2}+C r v=Q \\
C \frac{d r}{d t}=N .
\end{array}\right.
$$

Equations $(A)$ and $(B)$ are of the nature of an intrinsic form of statement of the dynamical conditions. If $T$ is a known function of $v$ and $s$, the first equation gives $v$ as a function of $s$. If, moreover, $N$ is known in terms of $v$ and $s$, the third equation, written in the form

$$
C v \frac{d r}{d s}=N
$$

yields $r$ as a function of $s$. And now the second equation determines $Q$ when $\kappa$ is a known function of $s$. If, on the other hand, $Q$ is a known function of $v$ and $s$, it determines $\kappa$ as a function of $s$. Here we have $\sqrt{s}$ given in intrinsic form,

$$
\kappa=\kappa(s) \text {. }
$$

Below is given the formula which expresses $\kappa$ in terms of the latitude and longitude of $\zeta$, and their derivatives. Hence the equation of $\mathbb{S}$ in terms of these coordinates can be derived.

An advantage of the foregoing form of the equations of motion, invariant as it is of a particular choice of the usual coördinates, lies in the directness with which qualitative questions relating to the motion can be handled. The applications to the top which are considered below illustrate this feature; $\mathrm{cf}$. in particular $\$ 6$, in which the fallacious explanation which is commonly given for the rising of the top with blunt peg on a rough table is exposed.

Furthermore, in the cases of greatest interest, the above dynamical equations suffice to determine the motion of the axis of the gyroscope without bringing in Euler's geometrical equations and, in particular, the third Eulerian angle, $\varphi$, with which we are not at all concerned.

The proof of the general theorem (Theorem II) is simple. It consists in resolving the angular momentum of the system,- - namely, the vector $(\sigma)$,-along three mutually perpendicular moving axes, viz. (i) the axis of the gyroscope; (ii) the tangent to $\sqrt{ }$, the path of $\zeta$; (iii) the normal to $\sqrt{ }$ in the tangent plane of the sphere. The vector form of the dynamical conditions governing the rotation is

$$
\frac{d(\sigma)}{d t}=\mathfrak{M}
$$


The bending, $\kappa$, presents itself on differentiating the unit vector which lies along the above normal.

\section{PROOF OF THE THEOREM}

We recall the definitions and the fundamental theorem of rigid dynamics with respect to rotation.

Let a particle of mass $m$ be moving with velocity represented by the vector $\mathfrak{b}$. Let $O$ be a point fixed in space, and let $\mathfrak{r}$ be the vector drawn from $O$ to the particle. The angular momentum of the particle with respect to $O$ is defined as the vector product:

$$
V \mathfrak{r}(m \mathfrak{v})
$$

For a system of discrete particles the angular momentum is defined as the sum of the angular momenta of the individual particles, and for a rigid body it is the corresponding integral. Let the latter be denoted by $(\sigma)$. Then

$$
(\sigma)=\lim _{n \rightarrow \infty} \sum_{i=1}^{n} V \mathfrak{r}_{i}\left(\mathfrak{v}_{i} \Delta m_{i}\right) .
$$

I,et a force represented by the vector $\mathfrak{F}$ act on $m$. The moment of $\mathfrak{F}$ with respect to $O$ is defined as the vector product of $\mathfrak{r}$ and $\mathfrak{F}$ :

$$
V \mathfrak{r} \mathfrak{F}
$$

If a system of forces, $\mathfrak{F}_{i}$, act at points, $P_{i}$, of the body, the moment of the system with respect to $O$ is defined as

$$
\mathfrak{M}=\sum_{i=1}^{n} \mathfrak{r}_{i} \mathfrak{F}_{i}
$$

where $\mathfrak{r}_{i}$ is the vector drawn from $O$ to $P_{i}$. In the case of a continuous distribution of force, the sum is to be replaced by the corresponding integral.

The fundamental theorem governing the rotation of a rigid body acted on by external, or applied, forces $\mathfrak{F}_{i}$ is

$$
\frac{d(\sigma)}{d t}=\mathfrak{M}
$$

where $\mathfrak{M}$ relates only to these forces, and not to any internal forces.

Case $\mathrm{I}$. We shall be concerned with this theorem in the case that a point of the axis of the gyroscope is fixed at $O$.

Case II. Let a rigid body be acted on by any applied forces, $\mathfrak{F}_{i}$, whatever. Let $(\sigma)$ be computed, not with reference to a point $O$ fixed in space, but with reference to the center of gravity, $G$, of the body; and let $\mathfrak{M}$ also be computed with 
respect to $G$ instead of $O$. Then equation (2) still holds, and governs the rotation of the body.

Case II is related to Case I as follows. Let a second body identical with the first have its center of gravity, $G^{\prime}$, fixed in space, and let it be acted on by the same relative forces; i. e., by forces of like intensities which are applied at corresponding points and act in corresponding directions, i. e., in the same directions relative to the second body. If, now, the two bodies were initially oriented alike and started alike, they will remain always oriented alike.

Since this latter body comes under Case I, it is sufficient for us to confine our attention to that case.

The vector $(\sigma)$ can be expressed in terms of the moments of inertia of the body about three principal axes of inertia through $O$ and the components of the vector angular velocity along these axes. Let three mutually perpendicular unit vectors be chosen as follows: $\mathfrak{a}$ shall be the vector drawn from $O$ to $\zeta$; shall be a

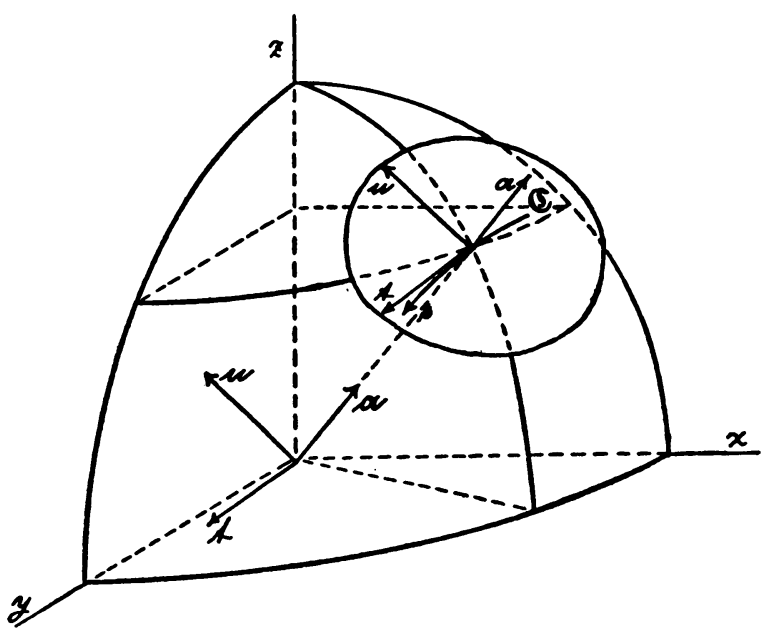

Fig. 1.

unit vector along the positive tangent to $\mathbb{S}$; and $\mathfrak{n}$ shall be a unit vector along the positive normal to $\mathfrak{C}$. Let $(\omega)$ be the vector angular velocity of the body about the instantaneous axis, and let $\omega_{t}, \omega_{n}, \omega_{a}$ be the components of $(\omega)$ along axes through $O$ parallel to or coincident with the above directions. Then, clearly, (for the definition of $r$ cf. foot note in $\$ 1$ )

$$
\omega_{\mathfrak{t}}=0, \quad \omega_{\mathfrak{n}}=v, \quad \omega_{\mathfrak{a}}=r .
$$

Moreover, the moments of inertia about these axes are:

$$
I_{\mathrm{t}}=A, \quad I_{\mathfrak{n}}=A, \quad I_{\mathrm{a}}=C .
$$


Now $(\sigma)$ has, by a well known theorem, the value

Hence

$$
(\sigma)=I_{\mathfrak{n}} \omega_{\mathfrak{n}} \mathfrak{n}+I_{\mathfrak{t}} \omega_{\mathfrak{t}} \mathfrak{t}+I_{\mathfrak{a}} \omega_{\mathfrak{a}} \mathfrak{a}
$$

$$
(\sigma)=A v \mathfrak{n}+C r \mathfrak{a} .
$$

From this equation it follows that

$$
\frac{d(\sigma)}{d t}=A v \frac{d \mathfrak{n}}{d t}+C r \frac{d \mathfrak{a}}{d t}+A \frac{d v}{d t} \mathfrak{n}+C \frac{d r}{d t} \mathfrak{a} .
$$

It is clear that

$$
\frac{d \mathfrak{a}}{d t}=v \mathrm{t}
$$

Since $\mathfrak{n}$ is a unit vector normal to the cone $\Omega$ at $\zeta$, we see that $d \mathfrak{n} / d s$ must be a vector parallel to $t$ and having its length numerically equal to the bending, $\kappa$. Scrutiny of the sign shows that

$$
\frac{d \mathfrak{n}}{d t}=\kappa v \mathrm{t}
$$

Hence, finally,

$$
\frac{d(\sigma)}{d t}=A v \frac{d v}{d s} \mathfrak{n}+\left(A \kappa v^{2}+C r v\right) \mathfrak{t}+C \frac{d r}{d t} \mathfrak{a} .
$$

Let the vector $\mathfrak{M}$ be resolved along the same three directions:

$$
\mathfrak{M}=\mathfrak{M}_{\mathfrak{n}} \mathfrak{n}+\mathfrak{M}_{\mathfrak{t}} \mathfrak{t}+\mathfrak{M}_{\mathfrak{a}} \mathfrak{a} .
$$

On equating the vectors (4) and (5) we obtain the three dynamical equations governing the rotation, in the form:

$$
\text { (6) } \quad A v \frac{d v}{d s}=\mathfrak{M}_{\mathfrak{n}}, \quad A \kappa v^{2}+C r v=\mathfrak{M}_{\mathrm{t}}, \quad C \frac{d r}{d t}=\mathfrak{M}_{\mathfrak{a}}
$$

'The case in which we are particularly interested is that in which the component of $\mathfrak{M}$ perpendicular to the axis of figure is due to a force $\mathfrak{F}$ at $\zeta$ tangent to the sphere. In terms of the above unit vectors, $\mathfrak{F}$ has the value:

$$
\mathfrak{F}=-Q \mathfrak{n}+T \mathfrak{t}
$$

Furthermore, $\mathfrak{M}_{\mathfrak{a}}=N$. We have, then, the three dynamical equations in the forn: 


$$
\begin{gathered}
A v \frac{d v}{d s}=T, \\
A \kappa v^{2}+C r v=Q, \\
C \frac{d r}{d t}=N .
\end{gathered}
$$$$
\text { q. e. d. }
$$

\section{The Bending, $\kappa$}

The bending of a cone has been defined in $\$ 1$ as the rate at which the tangent plane is turning when a point $\zeta$ on the generator at unit distance from the vertex moves with unit velocity.

Let

$$
\theta=f(\psi)
$$

be the equation of $\overleftarrow{\xi}, \S 1$, where $\theta$ and $\psi$ denote respectively the colatitude and the longitude of $\zeta$, and let $f(\psi)$, together with its first and second derivatives, be continuous. Let $P:(\theta, \psi)$ be an ordinary point of $\delta$. If we denote by $V$ the angle from the parallel of latitude through $P$ with the sense of the increasing $\psi$ to the tangent to $\mathcal{S}$ at $P$ with the sense of the increasing $s$, it is readily shown by means of infinitesimals that

$$
\kappa=\frac{d V}{d s}-\frac{d \psi}{d s} \cos \theta
$$

Since

$$
\tan V=\frac{d \theta}{d \psi \sin \theta}, \quad \text { or } \quad V=\tan ^{-1} \frac{\theta^{\prime}}{\psi^{\prime} \sin \theta}
$$

where accents shall denote differentiation with respect to $s$, and since the relation

$$
d s^{2}=d \theta^{2}+d \psi^{2} \sin ^{2} \theta
$$

gives

$$
\theta^{\prime 2}+\psi^{\prime 2} \sin ^{2} \theta=1, \quad \text { or } \quad \psi^{\prime}= \pm \csc \theta \sqrt{1-\theta^{\prime 2}}
$$

it is found that

$$
\kappa=\left(\psi^{\prime} \theta^{\prime \prime}-\psi^{\prime \prime} \theta^{\prime}\right) \sin \theta-\left(1+\theta^{\prime 2}\right) \psi^{\prime} \cos \theta
$$

Other formulas for $\kappa$ are:

$$
\pm \kappa=\frac{\theta^{\prime \prime}}{\sqrt{1-\theta^{\prime 2}}}-\sqrt{1-\theta^{\prime 2}} \cot \theta \text {; }
$$




$$
\kappa=\frac{\frac{d^{2} \theta}{d \psi^{2}} \sin \theta-2 \frac{d \theta^{2}}{d \psi^{2}} \cos \theta-\sin ^{2} \theta \cos \theta}{ \pm\left[\frac{d \theta^{2}}{d \psi^{2}}+\sin ^{2} \theta\right]^{\frac{3}{2}}} .
$$

In all these formulas, the upper sign is to be taken when $\psi$ increases with $s$. If $\boldsymbol{k}$ is given as a function of $s$, continuous together with its first derivative, formula (2) yields the following differential equation for determining $\theta$ :

$$
\frac{d^{2} \theta}{d s^{2}}=\left(1-\frac{d \theta^{2}}{d s^{2}}\right) \cot \theta \pm \kappa\left(1-\frac{d \theta^{2}}{d s^{2}}\right)^{\frac{1}{2}} .
$$

It is then possible to determine $\psi$ from the equation

$$
\frac{d \psi}{d s}= \pm \csc \theta\left(1-\frac{d \theta^{2}}{d s^{2}}\right)^{\frac{1}{2}}
$$

It is readily shown by infinitesimals that

$$
\mathbf{t}^{\prime \prime}=\kappa \mathfrak{n}-\mathfrak{a},
$$

where $\mathrm{t}^{\prime}=d \mathrm{t} / d s$. Now, the absolute value of $\mathrm{t}^{\prime}$ is equal numerically to the curvature, $K$, of $\mathbb{S}$ regarded as a twisted curve. Hence*

$$
K^{2}=\kappa^{2}+1
$$

Furthermore, the osculating plane of $\mathfrak{E}$ at $\zeta$ is determined by $t$ and $t^{\prime}$, both drawn from $\zeta$; and a unit vector, $\mathfrak{N}$, perpendicular to the osculating plane is given by the formula:

$$
\mathfrak{N}=\frac{1}{\sqrt{1+\kappa^{2}}} \mathfrak{n}+\frac{\kappa}{\sqrt{1+\kappa^{2}}} \mathfrak{a} .
$$

The torsion, $T$, of $\mathfrak{C}$ at $\zeta$ is equal numerically to the absolute value of $\mathfrak{R}^{\prime}$ On computing $\left|\mathfrak{N}^{\prime}\right|$ it is seen that $\dagger$

$$
\frac{d}{d s} \tan ^{-1} \kappa= \pm T
$$

the formula found by Professor Haskins.

Let $\subseteq$ be referred to a system of Cartesian axes through $O$, and let $\mathfrak{i}, \mathfrak{i}, \mathfrak{i}$ be unit vectors along these axes. Then

* Cf. Coolidge, Non-Euclidean Geometry, p. 189, Theorem 2.

† Ibid., p. 193. 


$$
\begin{aligned}
& \mathfrak{a}=x \mathfrak{i}+y \mathbf{i}+z \mathfrak{l}, \\
& \mathfrak{a}^{\prime}=x^{\prime} \mathfrak{i}+y^{\prime} \mathfrak{i}+z^{\prime} \mathfrak{l}=\mathfrak{t} \text {, } \\
& V a a^{\prime}=\left(y z^{\prime}-y^{\prime} z\right) \mathfrak{i}+\left(z x^{\prime}-z^{\prime} x\right) \mathfrak{i}+\left(x y^{\prime}-x^{\prime} y\right) \mathfrak{l}=\mathfrak{n} \text {. }
\end{aligned}
$$

We have seen that

$$
\mathfrak{n}^{\prime}=\kappa \mathrm{t} \text {. }
$$

Hence*

$$
\begin{aligned}
& y z^{\prime \prime}-y^{\prime \prime} z=\kappa x^{\prime}, \\
& x^{\prime \prime}-z^{\prime \prime} x=\kappa y^{\prime}, \\
& x y^{\prime \prime}-x^{\prime \prime} y=\kappa z^{\prime} .
\end{aligned}
$$

Since $|K|=\left|t^{\prime}\right|$ and

$$
\mathfrak{t}^{\prime}=x^{\prime \prime} \mathrm{i}+y^{\prime \prime} \mathrm{i}+z^{\prime \prime} \mathfrak{t}
$$

or

$$
K^{2}=x^{\prime \prime 2}+y^{\prime \prime 2}+z^{\prime \prime 2},
$$

we obtain, on squaring the equations (8) and adding, a new proof that

$$
K^{2}=\kappa^{2}+1
$$

If the cone $\Omega$ is a cone of revolution, it follows at once from the definition of the bending that $\kappa$ is constant. Let the axis of the cone pass through the north pole of the sphere, and let $\alpha$ be the semi-vertical angle of the cone. The equation of $\mathbb{E}$ is $\theta=\alpha$, and it follows from (2) that

$$
\pm \kappa=-\cot \alpha \text {. }
$$

Suppose, conversely, that $\alpha$ is constant. Then $\Omega$ must be a cone of revolution.

A first proof is given by ( 7$)$, for $\mathbb{C}$ is now a curve whose torsion vanishes identically. It is an elementary fact of differential geometry that such a curve is a plane curve. And since $\mathbb{E}$ lies on a sphere, it must be a circle. $\dagger$

Another proof follows at once from (4). Let $\alpha$ be determined by the conditions:

$$
\pm \kappa=-\cot \alpha, \quad 0<\alpha<\pi \text {. }
$$

Let the north pole be so chosen that (i) a point, $A$, of $(5$ will have the colatitude $\alpha$; and (ii) $\mathbb{S}$ will be tangent to the parallel of latitude at $A . \theta$ is determined

* Coolidge, loc. cit., p. 190.

$\dagger$ Professor Haskins applied his relation to this proof of the theorem. 
uniquely as a function of $s$ by (4) and the initial conditions:

$$
\theta_{0}=\alpha, \quad \theta_{0}^{\prime}=0 .
$$

One solution satisfying these conditions is

$$
\theta=\alpha ;
$$

and this is the only one. Hence the theorem is proved.

\section{Applications}

We will consider first some of the classical theorems about the gyroscope and show how their proofs follow immediately from the general or the restricted theorem of $\$ 1$.

(A) Steady Precession. Let the axis of the gyroscope describe a cone of revolution at a constant rate, $\dot{\psi}=\dot{\psi}_{0}>0$, and let $N=0$. Then $r=\nu$ (const.) $>0 ; T$ and $Q$ are given by Theorem $\mathrm{I}$ and are:

$$
T=0, \quad Q=C \nu v-A v^{2} \cot \alpha,
$$

where $\alpha$ is the semi-vertical angle of the cone; for

$$
\kappa=-\cot \alpha .
$$

The second equation (1) is the only relation which must hold between $v$, $\nu, \alpha$, and $Q$. In particular, $Q=0$ if

$$
C \nu=A v \cot \alpha .
$$

If
(i)
$\alpha>\cot ^{-1} \frac{C \nu}{A v}, \quad$ then $Q>0$

$$
\alpha<\cot ^{-1} \frac{C \nu}{A v}, \quad Q<0
$$

(ii)

In Case (i) $Q$ is directed away from the axis, i. e., along the outer normal of the cone; in Case (ii), the reverse.

Steady precession is also possible with $\dot{\psi}=\dot{\psi}_{0}<0, \nu>0$. Here, $\kappa=$ $\cot \alpha$, and

$$
Q=C \nu v+A v^{2} \cot \alpha .
$$

Thus $Q$ is always directed toward the axis of the cone.

(B) No Forces. This case is completely covered by equation (2) above, provided $v \neq 0$ :

$$
C \nu=A v \cot \alpha
$$


Any two of the three quantities $\nu, v, \alpha$ can be chosen at pleasure, subject merely to the restrictions:

$$
\nu>0, \quad v>0, \quad 0<\alpha<\frac{\pi}{2}
$$

and the third is then uniquely determined by (3). If $\alpha=\pi / 2$, then $\nu=0$ and $v$ is arbitrary.

Consider, for example, the earth, and suppose the sun, moon, and all other outside attracting matter were suddenly annihilated. What would be the effect on the motion of the axis, if the earth were rigid? A parallel to the axis through a fixed point describes a cone of revolution, of semi-vertical angle $23^{\circ}$ $27^{\prime}$, once in 25,800 years, and the angular velocity, $\nu$, corresponds to one revolution a day. The ratio $C / A$ is about $305 / 306$, and thus for present purposes is unity. Hence $\alpha$ can be computed from (3). It is found that the north pole would describe a circle of 21 inches diameter once a day (nearly).

Again, for the actual motion of the earth, the equation $\left(1^{\prime}\right)$ allows us to read off at once the value of the resultant couple exerted by the sun, the moon, and the other masses of the solar system.

$(C)$ The General Case, $\kappa=0$. Here $\zeta$ describes an arc of a great circle, with arbitrary velocity. Its motion along that circle is governed by the dynamical equations:

$$
A \frac{d v}{d t}=T, \quad C r v=Q, \quad C \frac{d r}{d t}=N
$$

The second of equations (4),

$$
Q=C r v,
$$

is the one most strongly emphasized in accounts of the gyroscope, when $v$ is constant and $N=0$. But (5) is seen to hold regardless of the values of $T$ and $N$. Thus it appears, for example, that sudden changes in $T$ and $N$ have no immediate effect on $Q$.

Again, if the motion of $\zeta$ in its path is prescribed; i. e., if $v=$ a given function of the time or the space; then $T$ will depend only on the time or the space, and not at all on $r$, which through proper choice of $N$ may be made any function of the time whatever, whose derivative is continuous.

(D) The Top. A top with fixed peg, $O$, which is executing a steady precession, is a particular case of the motion studied under $(A)$. Let $\alpha$ be the angle from the upward vertical through $O$ to the positive axis, $O \zeta$, of the top, and let $\psi$ be the longitude of the axis with reference to a vertical plane through $O$. Let the center of gravity lie on the positive axis, distant $h$ from $O$. Then $\mathfrak{F}$ lies in the meridian plane and is tilted downward. 


$$
|\mathfrak{F}|=M g h \sin \alpha= \pm Q, \quad v= \pm \dot{\psi}_{0} \sin \alpha, \quad-\kappa= \pm \cot \alpha,
$$

where the upper sign is to be chosen when $\dot{\psi}_{0}>0$; the lower, when $\dot{\psi}_{0}<0$. Thus we have from (1) the usual equation:

$$
A \dot{\psi}_{0}^{2} \cos \alpha-C \nu \dot{\psi}_{0}+M g h=0 .
$$

When $\alpha=\pi / 2$, (6) gives:

$$
\dot{\psi}_{0}=\frac{M g h}{C_{\nu}}
$$

For other values of $\alpha,(6)$ has a small positive root given approximately by (7), and a numerically large root algebraically slightly less than $C \nu /(A \cos \alpha)$.

(E) The Grape-Vine. Consider a top that is doing the grape vine.* Let it be mounted at its center of gravity, and let the only applied forces, aside from

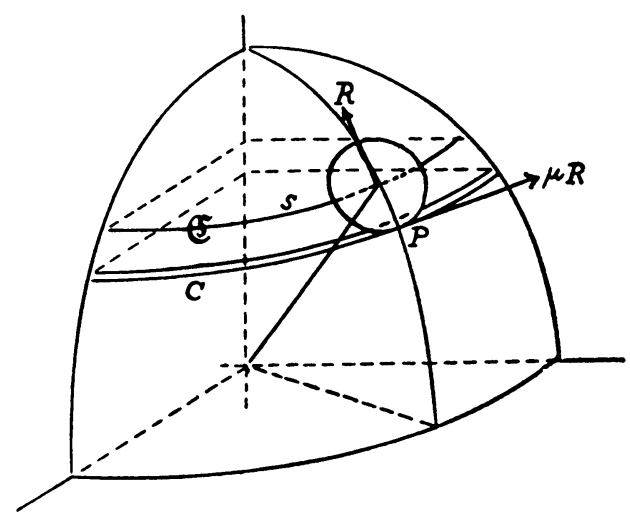

Fig. 2 .

the reaction at the peg, be the reaction of the constraint, the curve $C$. This latter force may be taken as consisting of a component $R$ normal to $\mathbb{S}$ and the axis of the top and passing through $\zeta$, and a component $\mu R$ tangential to $C$ at $P$ and hence parallel to the tangent to $\widetilde{S}$ at $\zeta$, and lying in the tangent plane of the sphere at $\zeta$. If we think of two forces equal and opposite to this latter component as applied at $\zeta$, the whole system of applied forces yields:

$$
T=\mu R, \quad Q=R, \quad N=-h \mu R,
$$

where $h$ is the radius of the axle of the top, or $P \zeta$.

We have assumed that initially $r$ is large and $v$ is positive and small, so that the axle is slipping on the constraint in the sense opposite to that of the motion of

* By this is meant the top of G. Sire, 1861. For the literature cf. Enzyklopädie der mathematischen Wissenschaften, vol. IV, p. 639. 
$\zeta$; moreover, that $\mathbb{E}$ and $C$ are related to each other in a one-to-one manner and continuously, so that, as $\zeta$ describes $\mathfrak{E}, P$ describes $C$ in like sense.

Under these initial conditions the axle will press against the constraint, as we have assumed it to do. For, if the constraint were not there, i. e., if the gyroscope were given the above initial motion with $\nu$ large and $v$ small, and left to itself, its axis would describe a very slender cone, since by (3) $\alpha$ would be small. Now, the constraint $C$ is in the way of such a motion of the axis, and the best the axis can do is to follow a curve $\sqrt{ }$ which is parallel to the constraint $C$.

So long as these conditions hold, the motion is governed by the equations of Theorem II, which here become:

$$
A \frac{d v}{d t}=\mu R, \quad A \kappa v^{2}+C r v=R, \quad C \frac{d r}{d t}=-h \mu R .
$$

From the first and third equations it appears that

$$
A h \frac{d v}{d t}+C \frac{d r}{d t}=0
$$

$$
A h v+C r=A h v_{0}+C r_{0} \text {. }
$$

Since $v$ is increasing and $r$ decreasing, there will come an instant when either (a) slipping ceases or $(b)$ the axle leaves the constraint.

In Case $(a)$, if $v_{1}, r_{1}$ are the values $v, r$ have when slipping ceases, then

$$
v_{1}=h r_{1},
$$

and hence, from (9),

$$
v_{1}=\frac{A h^{2} v_{0}+C r_{0} h}{A h^{2}+C}
$$

On eliminating $R$ and $r$ between the first two of equations (8), and (9), and setting $A h v_{0}+C r_{0}=A H$, we find:

$$
\frac{d v}{d s}=\mu(\kappa-h) v+\mu H .
$$

Since $\alpha$ is a function of $s$ determined by the constraint, (11) is a linear differential equation of the first order for determining $v$ as a function of $s$.

In Case (b), a first condition which must be realized, if the axle is to leave the constraint at an ordinary point $P^{\prime}$ of $C$, is that $R$ shall vanish. Hence, from the second of equations (8),

$$
-\kappa^{\prime}=\frac{C r^{\prime}}{A v^{\prime}}=\frac{H}{v^{\prime}} \cdots h .
$$


At this instant there are no forces acting, and the axis of the gyroscope would like to describe a cone of revolution, for which $\kappa=\kappa^{\prime}$. It will do so unless the constraint $C$ is in the way. Construct the cone of revolution and denote its intersection with the sphere by $\mathfrak{S}_{1}$. Also continue $C$ beyond $P^{\prime}$ and denote the path $\zeta$ would describe if the axle did not leave the constraint by $\mathfrak{G}_{2}$. If $\mathfrak{S}_{2}$, near $P^{\prime}$, lies inside $\mathfrak{G}_{1}$, the axle will leave the constraint; if $\mathfrak{G}_{2}$ lies outside of $\mathfrak{G}_{1}$ or coincides with it, the axle will not leave the constraint.

Suppose the curve $C$ comes to an end at a point $\vec{P}$, but that up to and inclusive of $\bar{P}$ is related in a one-to-one manner and continuously to its parallel curve $\mathfrak{E}$, and that the axle has not left the constraint, but reaches $\bar{P}$. Will the axle leave $C$ at $\bar{P}$ ? The answer can be given as in the previous case. Let $\bar{v}$ be the value of $v$ at $\bar{P}$, and let $\kappa$ be the bending of the cone the axis would now describe if the restraint were removed. By (3)

$$
-\bar{\kappa}=\cot \bar{\alpha}=\frac{C \bar{r}}{A \bar{v}} .
$$

On the other hand, if the axle clings to $\bar{P}$, the axis will describe a cone whose semi-vertical angle is

$$
\beta=\tan ^{-1} h \text {. }
$$

The condition that the axle should cling to $\bar{P}$ is obviously that $\bar{\alpha} \leqq \beta$, or

$$
A \bar{v} \leqq C h \bar{r} .
$$

I have discussed this problem at some length because it illustrates the ease with which quantitative results can be obtained by the theorem of $\S 1$.

(F) The Centrifugal Mill. The Griffin Mill described by Webster* is a particular case of the problem just discussed if gravity is neglected and the mill set in motion under the action of no other forces than the natural constraints. Since $\kappa$ is here constant, equation (11) can be integrated in closed form and the motion discussed in all detail.

Doubtless, however, to make the mill meet the needs of practice, the angular velocity $r$ would be controlled by a motor which would exert a couple just sufficient to offset the above couple $\mu h R$ and thus render $r$ constant: $r=\nu$. There would then be a further tangential force applied at $\zeta$, which would control the velocity $v$ and make it constant: $v=v_{0}$. The second equation of Theorem I would then determine $Q=R: \dagger$

$$
R=A \kappa v_{0}^{2}+C \nu v_{0},
$$

* Dynamics, 2d edition, Teubner, 1912, p. 273.

$\dagger$ The result agrees with Webster's formula (63), p. 274 . Webster resolves the angular velocity about the instantaneous axis along two oblique directions. 
where $\kappa=\cot \alpha$ and $\alpha$ is the angle the axis of the shaft makes with the downward vertical,-in the figure, the angle that $O \zeta$ makes with the upward vertical. $\kappa$ is here positive because $(5$ lies to the left of the positive tangent.

$(G)$ Friction at $\zeta$. It is interesting to discuss a case in which the force $\mathfrak{F}$ at $\zeta$ is always tangent to the path, $\mathfrak{S}$. Let the gyroscope be encased in a hollow sphere with centre at $O$ and radius slightly greater than 1 ; and let it carry a brush at $\zeta$ which rubs against the shell, thus exerting a constant force $f$ along the negative tangent at $\zeta$. Also, let $N=0$. The dynamical equations thus become:

$$
A v \frac{d v}{d s}=-f, \quad A \kappa v^{2}+C \nu v=0, \quad N=0
$$

From the first equation,

$$
\begin{gathered}
v^{2}=v_{0}^{2}-\frac{2 f}{A} s, \\
v=\frac{d s}{d t}=\sqrt{v_{0}^{2}-\frac{2 f}{A} s,} \\
t=\frac{A}{f}\left\{v_{0}-\sqrt{v_{0}^{2}-\frac{2 f}{A}} s\right\} .
\end{gathered}
$$

Hence $\zeta$ comes to rest after travelling a distance $s=A v_{0}^{2} / 2 f$ in time $t=A v_{0} / f$.

The curvature, $\kappa$, is given by the equation:

$$
\kappa=-\frac{C \nu}{A v}
$$

As $v$ steadily decreases, $\kappa$ increases numerically without limit, and the curve $\mathfrak{5}$ bends ever more sharply to the right. $\theta$ is given as a function of $s$ by (4), $\$ 3$.

It is to be observed that in this example the motion violates throughout the rule often given as the alpha and omega of the gyroscope,- to wit, that the axis moves at right angles to the forces of the couple, or the force $\mathfrak{F}$. Here, the point $\zeta$ always moves precisely in the line of the applied force, $\mathfrak{F}$.

One can imagine a case in which $\mathfrak{F}$ is just the reverse of that in the foregoing example. A centipede is forced to crawl on the inner surface of the shell and always draw the point $\zeta$ after him. The Devil sits at $\zeta$ and drives the centipede in the direction of the positive tangent. Here, $T=f>0, Q=0, N=0$, and a discussion similar to the above is in place. $\kappa$ now approaches 0 as $v$ increases without limit, and it is a matter of conjecture as to whether $\mathbb{S}$ has an asymptotic great circle. 


\section{The ToP with Fixed Peg}

The problem of the top with fixed peg, spinning with high angular velocity about its axis and acted on by gravity, but not by friction, is treated quantitatively by elementary methods, the latitude and longitude of $\zeta$ being obtained as functions of the time by means of quadratures. ${ }^{*}$ What these formulas fail to show is that the cone $\Re$ has the least number of inflectional tangent planes which is possible for any particular case of motion. This gap is easily filled by the foregoing methods, which furthermore yield an essential simplification in setting up the differential equations that govern the motion. It is found that the dynamical equations alone are sufficient to determine the motion of the axis, and thus all use of Euler's geometrical equations is avoided. In point of simplicity and directness, therefore, the following treatment would seem to yield a real gain.

Let $\theta$ and $\psi$ be respectively the colatitude and the longitude of $\zeta$, the ray $\theta=0$ being the upward vertical through the peg, $O$.

It is clear that the force $\mathfrak{F}$ is directed down the meridian through $\zeta$, and that its magnitude is $M g h \sin \theta$, where $h$ denotes the distance from $O$ to the center of gravity, assumed to lie on the same side of $O$ as $\zeta$. Hence the components $T$ and $Q$ have the values:

$$
T=M g h \sin \theta \frac{d \theta}{d s}, \quad Q=M g h \sin ^{2} \theta \frac{d \psi}{d s} .
$$

The value of $N$ is 0 , and so $r=\nu$.

From Theorem I we have:

$$
A v \frac{d v}{d s}=M g h \sin \theta \frac{d \theta}{d s}
$$

Integrating and setting

$$
u=\cos \theta, \quad a=\frac{2 M g h}{A},
$$

we obtain, on letting the subscript refer to initial values:

$$
v^{2}=v_{0}^{2}+a\left(u_{0}-u\right) \text {. }
$$

It is obvious that this is in substance the equation of energy.

A second integral of the dynamical equations is obtained by observing that, since $\mathfrak{M}$ is always horizontal, and since

$$
\frac{d(\sigma)}{d t}=\mathfrak{M},
$$

\footnotetext{
* For the classical treatment cf. Appell, Mécanique rationnelle, vol. 2, 2d edition, p. 195.
} 
the vertical component of $(\sigma)$ is constant.* Now, by $\S 2,(3)$,

$$
(\sigma)=A v \mathfrak{n}+C r \mathfrak{a} .
$$

The component in question has, therefore, the value:

$$
A v\left(\frac{d \psi}{d s} \sin \theta\right) \sin \theta+C \nu \cos \theta
$$

Hence we obtain as the integral in question:

$$
A v \frac{d \psi}{d s} \sin ^{2} \theta+C \nu \cos \theta=\text { const. }
$$

On setting

$$
\epsilon=\dot{\psi}_{0}\left(1-u_{0}^{2}\right), \quad \gamma=\frac{C \nu}{A}
$$

equation (3) hecomes:

$$
v\left(1-u^{2}\right) \frac{d \psi}{d s}=\epsilon+\gamma\left(u_{0}-u\right)
$$

Finally, there is the geometric relation arising from the equation

$$
d s^{2}=d \theta^{2}+d \psi^{2} \sin ^{2} \theta
$$

namely:

$$
\left(\frac{d \theta}{d s}\right)^{2}+\left(\frac{d \psi}{d s}\right)^{2}\left(1-u^{2}\right)=1
$$

From equations (i), (ii), and (iii) the two differential equations for $\theta$ and $\psi$ in the usual form follow at once: $\dagger$

$$
\begin{gathered}
\left(\frac{d u}{d t}\right)^{2}=\left(u_{0}-u\right)\left(u-u_{1}\right)(\lambda u+\mu), \\
\frac{d \psi}{d t}=\frac{\epsilon+\gamma\left(u_{0}-u\right)}{1-u^{2}}
\end{gathered}
$$

* This remark is standard for obtaining one of the differential equations of motion of the top. The form of $(\sigma)$ given just below leads immediately to this equation (3).

$\dagger$ The explicit formula obtained by elimination for $(d u / d t)^{2}$ involves a cubic polynomial:

$$
\frac{d u^{2}}{d t^{2}}=f(u)=\left(1-u^{2}\right)\left[v_{0}^{2}+a\left(u_{0}-u\right)\right]-\left[\epsilon+\gamma\left(u_{0}-u\right)\right]^{2} .
$$

Since $f(1) \leqq 0$ and $f(+\infty)=+\infty, f(u)$ has one root $\geqq+1$. Furthermore, $f(u)$ has a second root, $u_{0}$, between -1 and +1 . Finally, $f(-1) \leqq 0$. Hence, considering the character of the graph of such a cubic, we see that $f(u)$ must have its third root, $u_{1}$, in the interval $-1 \leqq u \leqq 1$. 
where $u_{1}, \lambda, \mu$ are constants such that $-1 \leqq u_{1} \leqq-1$, and $\lambda u+\mu \neq 0$ in the interval $-1<u<1$; moreover, $\dot{\theta}_{0}=0$. We prefer, however, to write these equations in the form:

$$
\begin{gathered}
\left(\frac{d \theta}{d s}\right)^{2}=\frac{\left(u_{0}-u\right)\left(u-u_{1}\right)(\lambda u+\mu)}{v^{2}\left(1-u^{2}\right)}, \\
\frac{d \psi}{d s}=\frac{\epsilon+\gamma\left(u_{0}-u\right)}{v\left(1-u^{2}\right)}
\end{gathered}
$$

where $v$ is given by (i), and $u=\cos \theta$. Equations (i) and (5) show that $s$ can be expressed as a function of $u$ by a quadrature. On substituting the inverse function for $u$ in (6), $\theta$ and $\psi$ can be expressed as functions of $s$ by means of quadratures.

We next compute $\kappa$. From (1) and (6),

$$
Q=\frac{M g h}{v}\left\{\epsilon+\gamma\left(u_{0}-u\right)\right\} \text {. }
$$

On substituting this value in the second equation of Theorem I and solving for $k$, we have:

$$
-\kappa=\frac{C \nu}{A v^{3}}\left\{v_{0}^{2}-\frac{M g h \epsilon}{C \nu}+\frac{a}{2}\left(u_{0}-u\right)\right\} .
$$

We now have all the material in hand for a detailed discussion of the character of $\mathcal{E}$ in each of the three cases indicated by Fig. 3 . In each case, $\dot{\theta}_{0}=0$. Moreover, in

Case I, $0<\dot{\psi}_{0}<c ; \quad$ Case II, $\dot{\psi}_{0}=0 ; \quad$ Case III, $-\frac{\gamma}{1-u_{0}}<\dot{\psi}_{0}<0$.

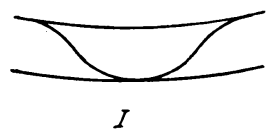

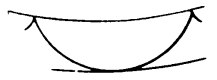

II

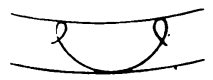

III

Fig. 3.

Here, $c$ is the value of $\dot{\psi}_{0}$ corresponding to a steady precession and is the numerically smaller of the two roots of $(6), \S 4$.

Case I. The initial value of $\alpha$ is given by the equation:

$$
-\kappa_{0}=\frac{C \nu}{A v_{0}^{3}}\left(v_{0}^{2}-\frac{M g h \epsilon}{C \nu}\right)=\frac{\gamma}{\sqrt{1-u_{0}^{2}}}\left\{\frac{1}{\dot{\psi}_{0}}-\frac{a}{2 \gamma} \cdot \frac{1}{\dot{\psi}_{0}^{2}}\right\} .
$$

For values of $\dot{\psi}_{0}$ in the interval $0 \leqq \dot{\psi}_{0} \leqq c$, the derivative

$$
\frac{d\left(-\kappa_{0}\right)}{d \dot{\psi}_{0}}=\frac{\gamma}{\dot{\psi}_{0}^{3} \sqrt{1-u_{0}^{2}}}\left\{\frac{a}{\gamma}-\dot{\psi}_{0}\right\}
$$


is positive; for, the value of $c$ is approximately, by $\$ 4,(7), a /(2 \gamma)$. Hence the derivative is positive for $\dot{\psi}_{0}=c$; and for smaller values of $\dot{\psi}_{0}$, the brace is still larger.

Thus a positive value of $\dot{\psi}_{0}$ less than $c$ gives rise to an algebraically larger value of $\kappa_{0}$ than the one which corresponds to $c$, and $\mathbb{E}$ begins to sink below the initial parallel of latitude, $\theta=\theta_{0}$. It follows, then, that $\theta$ increases, and hence $u_{1}<u_{0}$. Moreover, $u_{1}>-1$; for $f(-1)<0$.

From equations (6) it is now evident that $\sqrt{5}$ passes from the parallel of latitude $\theta=\theta_{0}$ to $\theta=\theta_{1}$, both $\theta$ and $\psi$ steadily increasing with $s$, and that this arc is tangent to each parallel at an extremity. Moreover, it is clear that the cone $\Omega$ must have an inflectional tangent plane at some point of this arc. What is not clear, however, from equations (6) is that there is only one such plane. This fact appears at once from (iv). The condition for an inflectional tangent plane is $\kappa=0$, or

$$
v_{0}^{2}-\frac{M g h \epsilon}{C \nu}+\frac{a}{2}\left(u_{0}-u\right)=0 .
$$

This equation must have at least one root, and it is seen at once that it has only one. Moreover, (8) gives the explicit determination of $u$ for this plane:

$$
u=u_{0}+\frac{2}{a}\left(v_{0}^{2}-\frac{M g h \epsilon}{C \nu}\right)
$$

The remainder of $\sqrt{ } S$ is found by reflection of the arc just obtained in the meridian plane through its lowest point, and periodic repetitions of the complete arc thus constructed.

Case II. Here, $\dot{\psi}_{0}=0$ and so $\epsilon=0$. The point $\zeta$ starts from rest, and since $v^{2}=a\left(u_{0}-u\right)$ from (i), it follows from (6) that $u_{1}<u_{0}$. Hence $\theta$ increases. Moreover, $u_{1}>-1$, as in Case I. The discussion is similar to that of Case I. It is clear from (iv) that $\kappa$ is never 0 or positive, and hence the cone $\Omega$ has no inflectional tangent planes. It has, of course, sharp edges at the cusps of $\mathfrak{(}$.

Case III. Here, $\dot{\psi}_{0}<0, \epsilon<0$. From (iv) it appears that $\kappa$ is initially negative, ${ }^{*}$ and hence $\theta$ begins by increasing. Thus $u_{1}<u_{0}$ and $\theta$ steadily increases till $\theta=\theta_{1}$. Consequently each term in the brace of (iv) is positive, and hence $\kappa$ is negative along the whole arc. Thus the cone $\Re$ has no inflectional tangent plane. From (6) it follows that, at the point $C$ where $(5$ meets the parallel of latitude $\theta=\theta_{1}, \mathbb{E}$ is tangent to this circle.

For values of $\dot{\psi}_{0}$ which are numerically small, $\epsilon$ will also be numerically small,

* We point out the inference to be drawn from this fact, namely, that near the initial point $(5$ lies below the great circle arc which is tangent to $\mathbb{S}$ at that point. 
and the second bracket in $f(u)$ will vanish for a value of $u$ lying in the interval $u_{1}<u<u_{0}$ :

$$
u^{\prime}=u_{0}+\frac{\epsilon}{\gamma}
$$

For this value of $u, d \psi / d s$ cnanges sign, and $\psi$ increases with $s$ in the interval $u_{1} \leqq u<u^{\prime}$.

On the other hand, if $\dot{\psi}_{0}$, and hence $\epsilon$, is numerically large, that bracket will never vanish, and $\psi$ will decrease forever. Obviously, in this case, too, $\alpha$ is always negative. The value of $\epsilon$ which separates these two cases is given by the condition that that bracket shall vanish for $u=u_{1}$. Consequently $u_{1}$ here $=-1$, and we have:

$$
\begin{gathered}
\epsilon+\gamma\left(u_{0}+1\right)=0, \quad \dot{\psi}_{0}\left(1-u_{0}^{2}\right)+\gamma\left(u_{0}+1\right)=0, \\
\dot{\psi}_{0}=-\frac{\gamma}{1-u_{0}} .
\end{gathered}
$$

Hence the second inequality for Case III.

If $\dot{\psi}_{0}=-\gamma /\left(1-u_{0}\right), \zeta$ passes through the south pole of the sphere.

Under Case I we demanded that $\dot{\psi}_{0}<c$. If $\dot{\psi}_{0}>c$, the curve $\sqrt{5}$ rises above the parallel of latitude $\theta=\theta_{0}$. For values of $\dot{\psi}_{0}$ only slightly greater than $c$, we get curves $\left(\mathcal{C}\right.$ like those in Case $\mathrm{I}$, the parallel $\theta=\theta_{0}$ playing the rôle of $\theta=\theta_{1}$ in Case I. As $\dot{\psi}_{0}$ continues to increase, we get curves $(5$ like those of Case III, the intermediate case being characterized by the fact that the root $u_{1}$ of $f(u)$ (which now is $>u_{0}$ ) must be the root of (8). The cases which correspond to Case III are those for which the second bracket in $f(u)$ has a root in the interval $u_{0}<u<u_{1} \quad$ When $\dot{\psi}_{0}$ has increased sufficiently, $d \dot{\psi} / d s$ is always $>0$. The two cases are separated by the value of $\dot{\psi}_{0}$ for which that bracket and $f(u)$ vanish simultaneously. Here, $u_{1}=1, \zeta$ goes through the north pole, and

$$
\begin{gathered}
\epsilon+\gamma\left(u_{0}-1\right)=0, \quad \dot{\psi}_{0}\left(1-u_{0}^{2}\right)+\gamma\left(u_{0}-1\right)=0, \\
\dot{\psi}_{0}=\frac{\gamma}{1+u_{0}} .
\end{gathered}
$$

When $\dot{\psi}_{0}>\gamma /\left(1+u_{0}\right), \kappa$ is always negative, and the path of $\zeta$ touches alternately the circles $\theta=\theta_{0}$ and $\theta=\theta_{1}$, which coalesce when $\dot{\psi}_{0}$ is equal to the larger root of $(6), \S 4$. A similar remark applies in Case III when $\dot{\psi}_{0}<-\gamma /\left(1-u_{0}\right)$.

Concerning Case III, we mention the one important fact which we have not been able to establish by the present methods, - namely, that $\psi_{1}>\psi_{0}$. Hadamard has, however, given an altogether simple proof of this relation by means of Cauchy's Integral Theorem; cf. B u 11 e t in des Sciences Mathém a t i q u e s, vol. 30 (1895), p. 228. 


\section{The Top with Blunt Rough Peg}

Consider a top with blunt peg spinning on a smooth horizontal plane. The peg is not approximately a conical point, but may be thought of as hemispherical, or as having the form of any surface of revolution which would correspond to a real top. The top is given a high angular velocity about its axis and released, or started with a moderate velocity of the axis, the peg touching a smooth horizontal table. We shall, furthermore, assume that the peg remains throughout the motion in contact with the table.

Since the forces acting, gravity and the reaction of the table, are both vertical, the horizontal motion of the center of gravity, $G$, will be uniform, and it is convenient to think of the initial velocity of $G$ as having no horizontal component. $G$ will then remain permanently in the vertical drawn through its initial position.

The cone $\Omega$ is here the family of rays drawn from an arbitrary fixed point, $O$, in space parallel to the axis of the top.

It is easy to set up the equations of motion, for the problem is closely akin to that of the top with fixed peg. The motion of the axis is similar to that of the earlier case. The axis falls and rises periodically, and executes a precession.* In particular, let the top be started so that we have Case I,-cf. Fig. $3, \S 5$.

A Rough Spot. Suppose the peg, in its course over the table, comes to a rough spot. Suppose, furthermore, that the point $P$ of the peg in contact with the

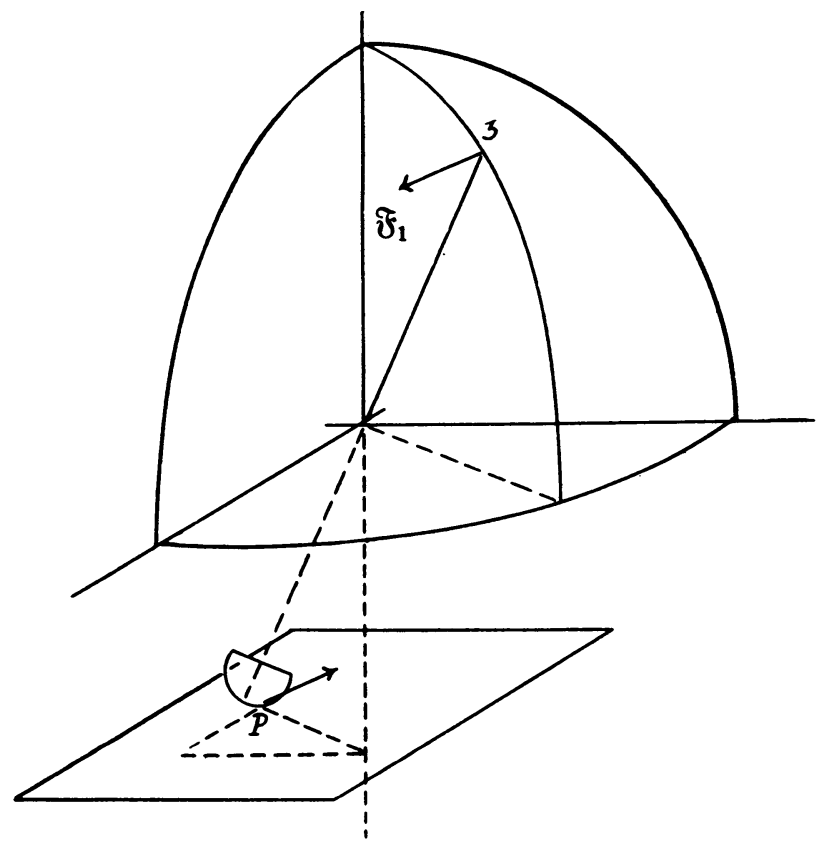

Fig. 4.

\footnotetext{
* Cf. Appell, loc. cit., p. 234.
} 
table is moving in a direction nearly or quite at right angles with the axis and toward the left of an observer looking from $G$ toward the peg. These conditions will be fulfilled since the motion of the axis is slow and the angular velocity of the top about its axis is high, if the peg be not too diminutive and the angle of the axis with the vertical reasonably large.

The horizontal force exerted by the table is here nearly or quite perpendicular to the axis of the top and toward the right of the observer. Let two forces, one equal and the other opposite to this force, be thought of as applied at the point $R$ of the axis nearest $P$.

To study the rotation of the actual top we will introduce a second top equal to the first, with its center of gravity fixed at $O$. It shall be oriented initially like the first and have initially the same (vector) angular velocity. Finally, it shall be acted on by the same relative forces as those which act on the actual top. It will, then, permanently be oriented like the actual top. The motion of the second top is governed by the theorems of $\$ 1$.

These new forces, the force of friction at $P$ and the two equal and opposite forces at $R$, give rise, so far as the rotation of the second top is concerned, to two new couples, namely: (a) a couple about the axis of the top, which diminishes the component of the angular velocity along that axis; its numerical value is small, and it has relatively small effect till considerable time has elapsed; (b) a couple represented by a force $\mathfrak{F}_{1}$ at $\zeta$ perpendicular to the axis of the top and nearly or

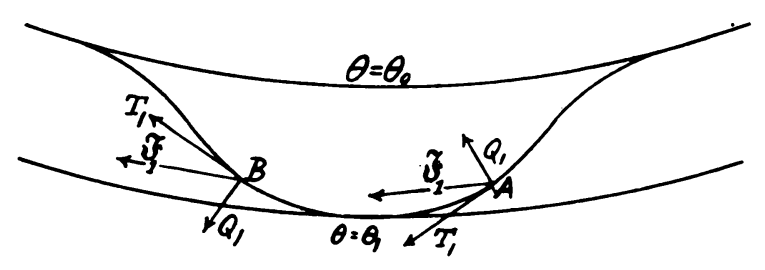

Fig. 5 .

quite perpendicular to the meridian plane through $\zeta$, its sense corresponding to that in which this plane is rotating. Let its components along the tangent and normal be $T_{1}$ and. $Q_{1}$, and let $T$ and $Q$ be the components of the force $F$ due to gravity.

Will the effect of the friction be to make the axis of the top rise or fall? We are now in a position to answer this question.

Case $A$. Suppose that, when the peg came to the rough spot, the axis was descending, $\zeta$ being at the point $A$ of $\mathfrak{E}$, Fig. 5. The value of $\kappa$ just before $A$ was reached was, by Theorem II, $\$ 1$ :

$$
\kappa=\frac{Q}{A v^{2}}-\frac{C r}{A v} .
$$


Just after $A$ is reached, $\kappa$ has the value

$$
\bar{\kappa}=\frac{Q+Q_{1}}{A v^{2}}-\frac{C r}{A v}=\kappa+\frac{Q_{1}}{A v^{2}} .
$$

There is no sudden change in the values of $v$ and $r$ near $A$; but the normal component of the applied force has jumped from $Q$ to $Q+Q_{1}$, and the bending of the cone $\Omega$ has thus experienced a discontinuity given by equation (2). Since $Q_{1}$ is negative, $\bar{\kappa}$ is less than $\kappa$, and so the new cone bears more sharply to the right (or less sharply to the left); thus the axis rises above the positions it would assume for like values of the longitude if the table were smooth.

Case $B$. Suppose, on the other hand, that, when the peg comes to the rough spot, the axis is ascending, $\zeta$ being at the point $B$ of $\mathfrak{C}$. Equations (1) and (2) still hold just before and just after $B$ is reached. Again there is a discontinuity in the bending. But here $Q_{1}$ is positive, $\bar{\kappa}$ is greater than $\kappa$, and the new cone bears more sharply to the left (or less sharply to the right). Hence the axis falls below the positions it would assume for like values of the longitude if the table were smooth.

The inference to be drawn from this example is that the rising of the axis of the top with rough peg is essentially a problem im Grossen. The explanations of the rising which depend on considerations im Kleinen are erroneous. ${ }^{*}$ It can be shown, it is true, that both in Case $A$ and in Case $B$ the angular momentum, i. e., the vector $(\sigma)$, rises. But the rising of $(\sigma)$ is neither a necessary nor a sufficient condition im Kleinen for the rising of the axis of the top.

Top with Fixed Peg, under Damping. Consider a gyroscope with a point $O$ of the axis fixed, which is acted on by no forces save the damping of the atmosphere and friction. Since the effect of the damping is here mainly to retard the angular velocity about the axis, we obtain a good approximation to the physical situation by assuming $\mathfrak{F}=0$, and $N$ negative and numerically small.

Since $T=0$, the velocity of the point $\zeta$ in its path, $\mathcal{G}$, is constant:

$$
\frac{d s}{d t}=v_{0}, \quad s=v_{0} t .
$$

Furthermore, since $Q=0$,

$$
-\kappa=\frac{C r}{A v_{0}},
$$

and the cone $\kappa$ steadily becomes flatter.

Finally,

$$
C \frac{d r}{d t}=N, \quad r=r_{0}-c t
$$

* Cf. Webster, loc. cit., p. 304. 
where $c=-N / C$. Hence

$$
r=r_{0}-\frac{c}{v_{0}} s
$$

and $\boldsymbol{k}$ is seen from (3) to be a linear function of $s$ :

$$
\kappa=-\frac{C}{A v_{0}}\left(r_{0}-\frac{c}{v_{0}} s\right) .
$$

On substituting this value of $\boldsymbol{\kappa}$ in the differential equation for $\theta$ as a tunction of $s, \S 3,(4)$, and solving, we have, with the further aid of equation (5), $\$ 3$, the analytic representation of $\sqrt{ }$ in this case.

\section{HARVARd UNIVERSITY,}

Cambridge, Mass. 